\begin{tabular}{|l|l|}
\hline $\begin{array}{l}\text { Instituto de } \\
\text { Geriatria e Gerontologia }\end{array}$ & $\begin{array}{l}\text { Pan American Journal of Aging Research } \\
\text { PAJAR, Porto Alegre, v. 8, p. 1-13, jan.-dez. } 2020 \\
\text { ISSN-L: 2357-9641 }\end{array}$ \\
\hline ittp://dx.doi.org/10.15448/2357-9641.2020.1.35971 & \\
\cline { 1 - 2 } &
\end{tabular}

ARTIGO ORIGINAL

\title{
Incontinência urinária em idosos da estratégia saúde da familia: prevalência e fatores de risco
}

\author{
Urinary incontinence in elderly family health strategy users: prevalence and risk factors \\ Incontinencia urinaria en ancianos estrategia de salud familiar: prevalencia y factores de riesgo
}

\author{
Vivian Ulrich ${ }^{1}$ \\ orcid.org/0000-0002-3893-711X \\ viviulrich@gmail.com
}

\section{Raquel Rousselet}

Farias $^{1}$

orcid.org/0000-0002-1870-8088 raquelfarias87@gmail.com

\section{Mara Regina Knorst ${ }^{1}$} orcid.org/0000-0001-8921-1469 mknorst@pucrs.br

\section{João Henrique Corrêa \\ Kanan ${ }^{2}$}

orcid.org/0000-0002-7768-9008

kananjhc@ufrgs.br

Thaís de Lima Resende ${ }^{1}$ orcid.org/0000-0002-8393-732X thaistr@pucrs.br

Recebido em: 14 out. 2019. Aprovado em: 9 dez. 2019 Publicado em: 03 agos.. 2020

\section{(c) (i)}

Artigo está licenciado sob forma de uma licença Creative Commons Atribuição 4.0 Internacional.

\section{Incontinência urinária em idosos de Porto Alegre \\ Resumo}

Objetivos: determinar a prevalência da incontinência urinária (IU) e a sua relação com aspectos sociodemográficos, antropométricos, funcionais e clínicos em idosos da Estratégia Saúde da Familia de Porto Alegre/RS (ESF/POA).

Métodos: estudo transversal analítico, coletado prospectivamente em amostra aleatória ( 30 unidades da ESF/POA). Foram coletados dados sociodemográficos, antropométricos e de saúde, sendo aplicados o Questionário Minessota de atividades físicas e de lazer, o Miniexame do Estado Mental, a Escala de Silhuetas de Stunkard (imagem corporal) e testes funcionais (Senta/Levanta, força de preensão manual e velocidade de caminhada).

Resultados: foram estudados 575 idosos $(68,9 \pm 7,1$ anos; mulheres= $64,35 \%$ ) dos quais $33,04 \%$ relataram IU (mulheres $=69,5 \%$ ). Foram estimados como fatores de risco para a IU: menor escore do Miniexame (OR=0,939; $\mathrm{P}=0,033 ; \mathrm{IC} 95 \%=0,887-$ $0,995)$; presença de prejuizo cognitivo $(O R=1,625 ; p=0,010 ; I C 95 \%=1,351-3,113)$; velocidade de caminhada mais lenta (OR=1,160; $p=0,016$; IC95\%=1,028-1,309); e menor escore no teste Senta/Levanta (OR=0,013; $p=0,874$; IC95\%=0,712 - 0,932). Quedas, atividade física, satisfação com a imagem corporal e ingestão medicamentosa não apresentaram associação significativa com a IU

Conclusão: nessa amostra, foi alta a prevalência da IU. Apresentar menor força de membros inferiores, menor velocidade de caminhada e prejuizo cognitivo foram identificados como fatores de risco para a incontinência, todos modificáveis. Palavras-chave: atenção primária à saúde, força muscular, caminhada, atividade física, imagem corporal, acidentes por quedas.

\section{Abstract}

Aims: to determine the prevalence of urinary incontinence and its relation with drug intake, cognition, functionality, falls, level of physical activity and body image in the elderly of the Family Health Strategy of Porto Alegre / RS (FHS/POA). Methods: a cross-sectional analytical study, prospectively collected in a random sample (30 units of FHS/POA). Sociodemographic, anthropometric and health data were collected, including on physical activity (Minnesota Physical and Leisure Activity Questionnaire), cognition (Mini Mental State Examination), body image satisfaction (Stunkard Silhouettes Scale) and functional ability (Chair Sit/Stand, hand grip strength and walking speed).

Results: a total of 575 elderly subjects $(68.9 \pm 7.1$ years; women $=64.35 \%)$ were studied; $33.04 \%$ reported urinary incontinence (women $=69.5 \%$ ). The following parameters were estimated as risk factors for incontinence: lower Mini Exam score $(\mathrm{OR}=0,939 ; \mathrm{p}=0,033 ; \mathrm{IC} 95 \%=0,887-0,995)$, presence of cognitive decline (OR= 1,625; $p=0,010 ;$ IC $95 \%=1,351-3,113)$, slower walking speed $(O R=1,160 ; p=0,016$ : $\mathrm{IC} 95 \%=1,028-1,309)$ and lower chair sit/stand score $(\mathrm{OR}=0,013 ; \mathrm{P}=0,874 ; \mathrm{IC} 95 \%=$ $0,712-0,932)$. Falls, physical activity, body image satisfaction and medication intake were not significantly associated with UI. 
Conclusion: in this sample, the prevalence of urinary incontinence was high and presenting weaker lower limb strength, worse mobility and cognition were identified as risk factors for incontinence, all modifiable.

Keywords: primary health care, muscle strength, walking, physical activity, body image, accidental falls.

\section{Resumen}

Objetivos: determinar la prevalencia de la incontinencia urinaria (IU) y su relación con aspectos socio demográficos, antropométricos, funcionales y clínicos en los ancianos de la Estrategia de Salud Familiar de Porto Alegre / RS (FHS / POA)

Métodos: Estudio analítico de corte transversal, recolectado prospectivamente en una muestra aleatoria (30 unidades FHS / POA). Se recopilaron datos socio demográficos, antropométricos y de salud, aplicando el Cuestionario de actividad física y de ocio de Minessota, el Mini examen del estado mental, la Escala de silueta de Stunkard (imagen corporal) y pruebas funcionales (Siéntate / Levantarse, fuerza de agarre). manual y velocidad de marcha).

Resultados: estudiamos a 575 ancianos (68.9 \pm 7.1 años; mujeres $=64.35 \%$ ) de los cuales $33.04 \%$ informaron IU (mujeres $=69.5 \%$ ). Los factores de riesgo para la IU se estimaron como: puntuación más baja en el Mini-Examen (OR = 0.939; $p=0.033 ; \mathrm{IC} 95 \%=0.887-0.955)$, presencia de deterioro cognitivo $(O R=1.625 ; p=0.010$; IC $95 \%=1.351-3.133)$, velocidad de marcha más lenta (OR = 1.160; $\mathrm{p}=0.016$; IC del $95 \%$ = 1.028-1.309), puntaje de prueba Siéntate / Levantarse más bajo (OR = 0.013; $p=0.874 ;$ IC del 95\% = 0.712-0.932). Las caídas, la actividad física, la satisfacción con la imagen corporal y la ingesta de medicamentos no se asociaron significativamente con la IU.

Conclusiones: en esta muestra, la prevalencia de IU fue alta, y la fuerza de las extremidades inferiores, la menor velocidad de marcha y el deterioro cognitivo se identificaron como factores de riesgo de incontinencia, todos modificables.

Palabras clave: atención primaria de salud, fuerza muscular, caminar actividad fisica, imagen corporal, accidentes por caídas.

\section{Introdução}

Envelhecer implica no declínio fisiológico das funções orgânicas e, em razão disso, uma maior probabilidade de surgimento de doenças crônicas, incapacitantes e evolutivas, que podem comprometer a autonomia das pessoas. Sendo assim, pessoas que vivem por mais tempo provavelmente irão sofrer de doenças crônicas não transmissiveis; dentre as quais está a incontinência urinária (IU). ${ }^{1}$ Esta é uma sindrome geriátrica frequente, ${ }^{3}$ cuja incidência aumenta linearmente com o avançar da idade, embora o seu aparecimento não faça parte do processo natural do envelhecer. ${ }^{2} \mathrm{~A}$ IU nos idosos, além de ter um grande peso econômico, pode ser um fator importante na institucionalização, na fragilidade, nas fraturas, na depressão e na pior qualidade de vida. ${ }^{3.4}$

O impacto da IU na terceira idade é significativo, ${ }^{4.5}$ assim sendo, desenvolver estratégias para o seu manejo adequado nesse grupo etário é fundamental em face da sua alta prevalência e do envelhecimento populacional acelerado que está acontecendo no País, onde a população idosa brasileira está crescendo em percentuais consideravelmente superiores ao da população total. ${ }^{6}$ Entre 2004 e 2012 esse crescimento foi na ordem de $27 \%$ e os censos demográficos trazem perspectivas de envelhecimento populacional ainda maiores em um futuro próximo. ${ }^{6}$ Esses dados são ainda mais preocupantes na capital do estado do Rio Grande do Sul: em Porto Alegre, 15,04\% da população é composta por idosos, sendo a capital com maior percentual de pessoas acima de 60 anos?

Por conseguinte, se faz necessário averiguar a prevalência e os fatores de risco da IU nos idosos de Porto Alegre, a fim de facilitar o planejamento das ações em saúde, permitindo o correto direcionamento das estratégias de promoção da saúde, prevenção das principais causas etiológicas e de tratamentos, com possibilidade real de melhora da qualidade de vida e de saúde dessa população. Dentre a população idosa da capital gaúcha, destaca-se a necessidade de investigar aquela servida pela Estratégia Saúde da Família (ESF), que, em geral, é implantada em "áreas periféricas, que apresentam maior dificuldade de acesso e maiores riscos de adoecer/morrer".

O presente estudo, portanto, foi desenvolvido com o objetivo de determinar a prevalência da IU em idosos da ESF de Porto Alegre/RS e a sua relação com dados sociodemográficos, ingestão medicamentosa, cognição, funcionalidade, quedas, nivel de atividade física e imagem corporal.

\section{Métodos}

O presente estudo transversal, prospectivo e analítico foi desenvolvido com dados do "Estudo epidemiológico e clínico dos idosos atendidos pela Estratégia de Saúde da Familia do municipio de Porto Alegre (EMISUS)", coletados entre março de 2011 e dezembro de 2012, após aprovação 
do Comitê de Ética em Pesquisa da Pontifícia Universidade Católica do Rio Grande do Sul (PUCRS) (Protocolo n. ${ }^{\circ}$ 10/04967) e da Secretaria Municipal de Saúde da Prefeitura do Município de Porto Alegre (Protocolo n. $\left.{ }^{\circ} 001.021434 .10 .7\right) .^{9}$

Para acesso à amostra aleatória da população de idosos cadastrados na ESF, foram sorteadas 30 equipes da ESF, de forma estratificada por gerência distrital, sendo selecionadas em torno de $30 \%$ das equipes de cada gerência. ${ }^{9}$ Os critérios de inclusão foram: idade igual ou superior a 60 anos e estarem cadastrados na ESF. Foram excluídos os individuos sem condições clínicas de ir ao Hospital São Lucas (HSL) da PUCRS para a avaliação multiprofissional. ${ }^{9}$ Entretanto, o número de idosos que não conseguiram ir até o hospital foi irrelevante, posto que aqueles que não conseguiam caminhar até a sua unidade de saúde, onde eram aguardados pelo transporte fornecido pelo grupo de pesquisa, foram buscados em seu domicilio.

$A$ aquisição dos dados foi realizada em duas etapas: (1) triagem e coleta de dados gerais, onde, em visita domiciliar, os agentes comunitários de saúde entrevistaram os idosos e aplicaram um questionário (Questionário de
Avaliação Global do Idoso) contendo questões relacionadas a aspectos sociodemográficos, econômicos e sociais, morbidade referida e comportamentos que interferem na saúde; e (2) avaliação de aspectos clínicos, nutricionais e físicos dos idosos realizada no HSL por equipe multidisciplinar especializada. Para essa etapa da coleta foi fornecido transporte gratuito para os participantes, seus cuidadores e agentes comunitários de saúde a partir da sua unidade de saúde ou de sua moradia. ${ }^{9}$

A descrição das variáveis do estudo, os instrumentos utilizados e o local da coleta podem ser vistos na Tabela 1. Foram utilizadas as seguintes variáveis: idade; faixa etária; estado civil; escolaridade; peso; altura; indice de massa corporal (IMC); tempo para caminhar $10 \mathrm{~m}$; Teste do Senta/ Levanta; força de preensão manual; cognição (Miniexame do Estado Mental - MEEM); satisfação com a imagem corporal (SIC; Escala de Silhuetas de Stunkard); atividade física (Questionário de Minessota); relato de perda urinária e quedas; e ingestão medicamentosa e polifarmácia ( $\geq 4$ medicamentos utilizados concomitantemente).

TABELA 1 - Descrição das variáveis do estudo, categorias, instrumentos e locais da coleta

\begin{tabular}{|c|c|c|c|c|}
\hline \multirow{2}{*}{ VARIÁVEIS } & \multirow{2}{*}{ CATEGORIAS } & & \multicolumn{2}{|l|}{ COLETA } \\
\hline & & & Instrumento & Local \\
\hline \multirow{5}{*}{ Sociodemográficas } & Idade (anos) & Não & QAGI & Domicilio \\
\hline & Faixa etária & $\begin{array}{l}60 \text { a } 69 \text { anos; } 70 \text { a } 79 \\
\text { anos; } \geq 80 \text { anos }\end{array}$ & QAGI & Domicilio \\
\hline & Escolaridade & $\begin{array}{l}\text { Analfabetos; baixa; } \\
\text { média; alta escolaridade }\end{array}$ & QAGI & Domicilio \\
\hline & Estado civil & $\begin{array}{l}\text { Convive maritalmente / } \\
\text { não convive maritalmente }\end{array}$ & QAGI & Domicilio \\
\hline & Nivel de atividade física & $\begin{array}{l}\text { Total; leve; moderada; } \\
\text { intensa }\end{array}$ & $\begin{array}{l}\text { Questionário } \\
\text { Minnesota }\end{array}$ & HSL \\
\hline \multirow{3}{*}{ Antropométricas } & Peso & Não & Balança Welmy® & HSL \\
\hline & Altura & Não & Fita métrica & HSL \\
\hline & IMC & $\begin{array}{l}\text { Baixo peso; eutrófico; } \\
\text { sobrepeso/obesidade }\end{array}$ & $\begin{array}{l}\text { Quetelet } \\
(\text { (IMC= peso/altura²) }\end{array}$ & HSL \\
\hline
\end{tabular}




\begin{tabular}{|c|c|c|c|c|}
\hline \multirow{2}{*}{ VARIÁVEIS } & \multirow{2}{*}{ CATEGORIAS } & & \multicolumn{2}{|l|}{ COLETA } \\
\hline & & & Instrumento & Local \\
\hline \multirow{3}{*}{ Funcionais } & $\begin{array}{l}\text { Tempo (s) para } \\
\text { caminhar } 10 \text { metros }\end{array}$ & Não & Cronômetro & HSL \\
\hline & Teste do Senta/Levanta & Não & Cronômetro & HSL \\
\hline & $\begin{array}{l}\text { Força de preensão } \\
\text { manual }\end{array}$ & Não & $\begin{array}{l}\text { Dinamômetro } \\
\text { Crow }{ }^{\circledR}\end{array}$ & HSL \\
\hline \multirow{9}{*}{ Clinicas } & Cognição & Não & MEEM & \\
\hline & $\begin{array}{l}\text { Presença de prejuizo } \\
\text { cognitivo }\end{array}$ & Sim / Não & MEEM & HSL \\
\hline & $\begin{array}{l}\text { Satisfação com a } \\
\text { imagem corporal }\end{array}$ & Sim / Não & Escala de Stunkard & HSL \\
\hline & Incontinência urinária & Sim / Não & QAGI & HSL \\
\hline & $\begin{array}{l}\text { Relato de quedas no } \\
\text { último ano }\end{array}$ & Sim / Não & QAGI & HSL \\
\hline & $\begin{array}{l}\text { № de vezes que caiu } \\
\text { no último ano }\end{array}$ & Uma; duas; três & QAGI & HSL \\
\hline & Fratura devido a queda & Sim / Não & QAGI & HSL \\
\hline & Utiliza medicamentos & Sim / Não & QAGI & HSL \\
\hline & $\begin{array}{l}\text { No. medicamentos } \\
\text { utilizados }\end{array}$ & Nenhum; de 1 a $3 ; \geq 4$ & QAGI & HSL \\
\hline
\end{tabular}

QAGI: Questionário de Avaliação Global do Idoso; HSL: Hospital São Lucas da Pontifícia Universidade Católica do Rio Grande do Sul; Questionário Minnesota: Questionário Minnesota de Atividade Física, Esporte e Lazer; IMC: indice de massa corporal; MEEM: Miniexame do Estado Mental; Escala de Stunkard: Escala de Silhuetas de Stunkard.

Para a mensuração do peso e da estatura, após descartadas vestes e objetos pesados, o idoso foi posicionado sem calçados em ortostase no centro de uma balança Filizola com estadiômetro acoplado. Em termos do IMC, os idosos que apresentaram valores até $22 \mathrm{~kg} / \mathrm{m} 2$ foram considerados em situação de baixo peso; entre $22 \mathrm{~kg} / \mathrm{m} 2$ e $27 \mathrm{~kg} / \mathrm{m} 2$ como eutróficos; e acima de $27 \mathrm{~kg} / \mathrm{m} 2$, em estado de obesidade. ${ }^{10}$

O tempo para caminhar $10 \mathrm{~m}$ foi mensurado em espaço plano e reto, sem outras pessoas que não o(a) participante sendo testado(a) e registrado para o centésimo de segundo mais próximo.11

O Teste de Senta/Levanta foi aplicado com o participante sentado em uma cadeira, com as costas eretas, os pés apoiados no solo e os braços cruzados e apoiados no tórax. Ao sinal "Atenção! Já!" o avaliado levantava-se, ficava totalmente em pé e, então, retornava à posição completamente sentado. O participante foi encorajado a levantar e sentar-se completamente o maior número de vezes possiveis em 30 segundos..$^{12}$

As medidas da força de preensão manual do membro superior dominante foram realizadas com o indivíduo sentado em uma cadeira, com o tronco ereto, pés apoiados no chão, ombro em adução e rotação neutra, cotovelo flexionado a $90^{\circ}$, com o antebraço em posição neutra em suspensão no ar e suportado pelo avaliador. Três medidas foram realizadas com um intervalo de 60 segundos entre elas, sendo utilizada a maior delas. ${ }^{13}$

Os pontos de corte adotados para o MEEM 
foram os seguintes: 13 para analfabetos e analfabetos funcionais, 18 para escolaridade baixa/média, e 26 para alta escolaridade. ${ }^{14}$

A escala de Stunkard foi aplicada em entrevista individual onde o idoso foi orientado a apontar duas figuras corporais, sendo que a primeira representava a sua silhueta atual e a outra a que desejaria ter; para definição da insatisfação corporal foi utilizada a discrepância entre essas medidas. ${ }^{15}$

No presente estudo foram utilizados os totais de minutos por semana que foram despendidos em atividades consideradas leves, moderadas ou intensas em termos do dispêndio energético, bem como a soma de todos os minutos despendidos nos três niveis de atividade física, ou seja, o escore total do Questionário Minnesota de Atividade Física, Esporte e Lazer. Esse instrumento é composto por duas baterias de itens; uma delas investiga a prática de esportes ativos e de exercícios físicos e a outra, a realização de tarefas domésticas. ${ }^{16}$

A perda urinária foi avaliada pela questão "Você perde urina?". Não foi utilizado teste diagnóstico especifico para a incontinência, a qual, também, não foi classificada em categorias conforme os tipos de IU, posto que essa classificação exigiria conhecimento clínico. Da mesma forma, perguntas dicotômicas foram feitas em relação à ocorrência de quedas, uso de medicamentos etc., todas baseadas no autorrelato.

Os dados foram analisados no programa Statistical Package for Social Sciences, versão 20.0 (SPSS, Chicago, IL, EUA) para Windows, tendo sido adotado o nivel de significância de 5\%. O estudo da distribuição de dados das variáveis contínuas ocorreu pelo teste de Kolmogorov-Smirnov.

Para a análise bivariada entre variáveis categóricas foi utilizado o teste Qui-quadrado de Pearson $\left(\chi^{2}\right)$ no qual, nas tabelas de contingência $2 \times 2$, foi utilizada a correção de continuidade, com estimativa da medida de efeito, o Odds ratio (OR) bruto, com intervalo de confiança de 95\% (IC95\%). Para as variáveis contínuas, quando a comparação ocorreu entre dois grupos independentes foram aplicados os testes de t-Student ou de Mann Whitney U.

Dada a distribuição binária do desfecho "Perda Urinária", foi aplicada a Regressão Logística (método Backward condicional a partir do modelo saturado) a todas as variáveis com valores de $\mathrm{p}<0,200$ na análise bivariada. A associação foi avaliada com o teste da razão de máxima verossimilhança (likelihood-ratio test - 2LL ou -2log) e, para avaliar a qualidade do ajuste do modelo final de regressão logística, foram utilizados os estimadores de $\mathrm{R}^{2}$ de Nagelkerk e Hosmer-Lemeshow. A probabilidade de entrada gradual das variáveis ao modelo foi de 0,05 e para a remoção de 0,10. A significância foi de 0,05 para o máximo de 20 interações. Os niveis de significância inferiores a 0,01 foram considerados significativos com base no critério de Bonferroni.

\section{Resultados}

O banco de dados original continha 587 casos, no entanto, a amostra analisada foi composta por 575 investigados, posto que foram considerados apenas os casos que apresentaram respostas válidas para o fator de estudo - relato de perda urinária. A amostra foi composta, em sua maioria, por mulheres $(64,3 \%)$, com idades variando entre 60 e 103 anos, 63,8\% sem convívio marital, 91,6\% analfabetas, $70,4 \%$ sem comprometimento cognitivo (MEEM), e 57,8\% apresentando sobrepeso/obesidade.

Conforme pode ser visto na Tabela 2, a idade mais elevada mostrou-se como um fator de risco para a perda urinária, uma vez que os investigados mais velhos apresentaram 1,028 vezes mais chance de relatar perda de urina, quando comparados aos mais jovens. Esse resultado se mostrou ainda mais claro na comparação das faixas etárias, onde o grupo com 80 anos ou mais apresentou 2,196 vezes mais chance de relatar perda de urina do que o grupo até 69 anos. 
TABELA 2 - Características sociodemográficas da amostra, segundo o relato de perda urinária

\begin{tabular}{|c|c|c|c|c|c|}
\hline \multirow{2}{*}{ VARIÁVEIS } & \multicolumn{2}{|c|}{ PERDA URINÁRIA } & \multirow{2}{*}{$\mathbf{p}^{\varepsilon}$} & \multicolumn{2}{|c|}{ ODDS RATIO BRUTO } \\
\hline & $\operatorname{Sim}(n=190)$ & Não $(n=385)$ & & OR & $\mathrm{IC} 95 \%$ \\
\hline \multicolumn{6}{|l|}{ Sexo * } \\
\hline Masculino & $58(30,5)$ & $147(38,2)$ & $0,071^{5}$ & 1,0 & \\
\hline Feminino & $132(69.5)$ & $238(61,8)$ & & 1,115 & $0,994-1,250$ \\
\hline \multicolumn{6}{|l|}{ Idade (anos) } \\
\hline Média $\pm D P$ & $70,1 \pm 7,6$ & $68,4 \pm 6,9$ & $0,007^{€}$ & & \\
\hline Amplitude & $60-100,2$ & $60-103,8$ & & 1,028 & $1,004-1,053$ \\
\hline \multicolumn{6}{|l|}{ Faixa etária * } \\
\hline Até 69 anos & $107(56,3)$ & $255(66,2)$ & $0,022^{\S}$ & 1,0 & \\
\hline De 70 a 79 & $60(31,6)$ & $105(27,3)$ & & 1,362 & $0,9232,010$ \\
\hline 80 anos ou mais & $23(12,1)$ & $25(6,5)$ & & 2,196 & $1,192-4,034$ \\
\hline \multicolumn{6}{|l|}{ Estado civil * } \\
\hline Convive maritalmente & $63(33,2)$ & $145(37,7)$ & $0,290^{\S}$ & 1,0 & \\
\hline Não convive maritalmente & $127(66,8)$ & $240(62,3)$ & & 1,066 & $0,949-1,198$ \\
\hline \multicolumn{6}{|l|}{ Escolaridade * } \\
\hline Analfabeto & $179(94,2)$ & $347(90,4)$ & $0,485^{\varsigma}$ & 0,872 & $0,828-2,529$ \\
\hline Baixa escolaridade & $11(5,8)$ & $37(9,6)$ & & 1,0 & \\
\hline \multicolumn{6}{|l|}{ Peso (kg) } \\
\hline Média $\pm D P$ & $70,1 \pm 13,8$ & $70,3 \pm 14,3$ & $0,909^{*}$ & & \\
\hline Amplitude & $42,0-116,8$ & $32,4-122,1$ & & 0,999 & $0,987-1,012$ \\
\hline \multicolumn{6}{|l|}{ Altura $(\mathrm{cm})$} \\
\hline $\begin{array}{l}\text { Média } \pm \text { DP } \\
\text { Amplitude }\end{array}$ & $\begin{array}{l}1,55 \pm 0,09 \\
1,32-1,79\end{array}$ & $\begin{array}{l}1,57 \pm 0,05 \\
1,35-1,80\end{array}$ & $0,001^{\sharp}$ & 0,034 & $0,004-0,280$ \\
\hline \multicolumn{6}{|l|}{$\mathrm{IMC}\left(\mathrm{kg} / \mathrm{m}^{2}\right)$} \\
\hline Amplitude & $\begin{array}{l}29,3 \pm 5,8 \\
17,4-54,1\end{array}$ & $\begin{array}{r}28,3 \pm 5,5 \\
13,6-49,2\end{array}$ & $0,052^{7}$ & 1,032 & $1,000-1,065$ \\
\hline \multicolumn{6}{|l|}{ Categoria do IMC * } \\
\hline Baixo peso $(I M C<22)$ & $11(6,4)$ & $38(10,0)$ & & 1,0 & \\
\hline Eutrófico $(22-27)$ & $58(33.5)$ & $126(33,2)$ & $0,361^{\S}$ & 0,874 & $0,602-1,074$ \\
\hline $\begin{array}{l}\text { Sobrepeso/obesidade } \\
\text { (IMC>27) }\end{array}$ & $104(60,1)$ & $215(56,7)$ & & 0,955 & $0,844-1,112$ \\
\hline
\end{tabular}

£: Nivel mínimo de significância para a análise bivariada; OR: Odds Ratio; IC95\% Intervalo de confiança de 95\% para o OR; ": dados apresentados na forma de n(\%); DP: desvio padrão da média; IMC: indice de massa corporal; $\S$ Teste Qui-quadrado de Pearson; €: Teste t-Student para grupos independentes assumindo heterogeneidade de variâncias; ¥: Teste t-Student para grupos independentes assumindo homogeneidade de variâncias; Baixo peso: IMC<22; Eutrófico: IMC entre 22 e 27; Sobrepeso / obesidade: IMC>27. 
A baixa estatura (altura) e um IMC mais alto também se apresentaram como fatores de risco para o relato de perda urinária (Tabela 2). As demais variáveis apresentadas na Tabela 2 (estado civil, escolaridade, peso e categorias do IMC), não apresentaram resultados significativos quando comparadas ao relato de perda urinária.

Em termos de funcionalidade (Tabela 3), crescem as chances de haver relato de perda urinária quanto maior for o tempo gasto para caminhar $10 \mathrm{~m}$ (velocidade de caminhada mais lenta), bem como sentar-se e levantar-se um menor número de vezes. Não foram detectadas associações significativas entre o relato de perda urinária e força de preensão palmar, satisfação com a imagem corporal ou qualquer um dos quatro escores da medida de atividade física (Tabela 3).

TABELA 3 - Características funcionais, satisfação com a imagem corporal e nível de atividade física da amostra, segundo o relato de perda urinária.

\begin{tabular}{|c|c|c|c|c|c|}
\hline \multirow{2}{*}{ VARIÁVEIS } & \multicolumn{2}{|c|}{ PERDA URINÁRIA } & \multirow{2}{*}{$\mathbf{p}^{\mathbf{E}}$} & \multicolumn{2}{|c|}{ ODDS RATIO BRUTO } \\
\hline & $\operatorname{Sim}(n=190)$ & Não (n=385) & & OR & $\mathrm{IC}_{95 \%}$ \\
\hline \multicolumn{6}{|c|}{ Tempo para caminhar 10 m (s) } \\
\hline Média $\pm D P$ & $7,2 \pm 2,2$ & $6,4 \pm 1,9$ & $<0,001^{¥}$ & 1,218 & $1,109-1,338$ \\
\hline Amplitude & $4,0-16,0$ & $3,0-20,0$ & & & \\
\hline \multicolumn{6}{|c|}{ Teste do Senta/levanta (no vezes/30s) } \\
\hline Média $\pm D P$ & $8,1 \pm 3,1$ & $9,2 \pm 3,1$ & $<0,001^{¥}$ & 0,887 & $0,831-0,947$ \\
\hline Amplitude & $1,0-18,0$ & $1,0-23,0$ & & & \\
\hline
\end{tabular}

\section{Força de preensão manual (Kgm)}

$\begin{array}{llllll}\text { Média } \pm D P & 63,6 \pm 358,4 & 89,6 \pm 619,4 & 0,609^{7} & 0,887 & 0,685-1,193 \\ \text { Amplitude } & 5,7-3354,7,0 & 5,2-6674,0 & & \end{array}$

Satisfação com imagem corporal *

\begin{tabular}{|c|c|c|c|c|c|}
\hline Sim & $51(29,8)$ & $111(31,6)$ & $0,677^{\S}$ & 1,0 & \\
\hline Não & $120(70,2)$ & $240(68,4)$ & & 1,028 & $0,905-1,167$ \\
\hline
\end{tabular}

Atividade Física Total (minutos/semana) **

\begin{tabular}{|c|c|c|c|c|c|}
\hline Mediana & 2398,0 & 3495,0 & $0,328^{\mathbb{T}}$ & 0,804 & $0,613-1,864$ \\
\hline $1^{\circ}-3^{\circ}$ Quartil & $1209,0-4459,0$ & $1921,5-7325,0$ & & & \\
\hline
\end{tabular}

Atividade Física Leve (minutos/semana) **

\begin{tabular}{|c|c|c|c|c|c|}
\hline Mediana & 2150,0 & 2296,0 & $0,361^{\text {TI }}$ & 0,877 & $0,578-2,008$ \\
\hline $1^{\circ}-3^{\circ}$ Quartil & $815,0-3451,0$ & $270,0-4180,0$ & & & \\
\hline
\end{tabular}

Atividade Física Moderada (minutos/semana) **

\begin{tabular}{|c|c|c|c|c|c|}
\hline Mediana & 1278,0 & 1600,0 & $0,690^{\text {वा }}$ & 0,933 & $0,623-1,479$ \\
\hline $1^{\circ}-3^{\circ}$ Quartil & $387,0-2503,0$ & $658,0-3504,0$ & & & \\
\hline
\end{tabular}

Atividade Fisica Intensa (minutos/semana) **

$\begin{array}{lccccc}\text { Mediana } & 408,0 & 973,0 & 0,656^{\mathbb{T}} & 1,112 & 0,822-2,518 \\ 1^{\circ}-3^{\circ} \text { Quartil } & 191,0-1086,0 & 432,0-2446,0 & & & \end{array}$

£: Nivel mínimo de significância para a análise bivariada; OR: Odds Ratio; IC95\% Intervalo de confiança 95\% para o OR; DP: desvio padrão da média; *: dados apresentados na forma de n(\%); *: dados apresentados na forma de $n(\%) ;{ }^{*}$ : Atividade física mensurada com o Questionário Minnesota de atividades físicas e de lazer; ¥: Teste t-Student para grupos independentes assumindo homogeneidade de variâncias; §: Teste Qui-quadrado de Pearson; T:Teste de Mann Whitney. 
Na Tabela 4 são apresentadas as caracteristicas clínicas da amostra, onde se observa que, enquanto um escore mais elevado no MEEM mostrou-se um fator protetor, ter um histórico de quedas, bem como relatar a ocorrência de quedas nos últimos 12 meses foram estimados como potenciais fatores de risco para o relato de perda urinária. Foram detectadas relações de independência entre o relato de perda urinária e a ingestão medicamentosa, bem como o número de medicamentos utilizados (Tabela 4).

TABELA 4 - Características clínicas da amostra, segundo o relato de perda urinária.

\begin{tabular}{|c|c|c|c|c|c|}
\hline \multirow{2}{*}{ VARIÁVEIS } & \multicolumn{2}{|c|}{ PERDA URINÁRIA } & \multirow{2}{*}{$\mathbf{p}^{\mathfrak{E}}$} & \multicolumn{2}{|c|}{ ODDS RATIO BRUTO } \\
\hline & $\operatorname{Sim}(n=190)$ & Não (n=385) & & OR & IC95\% \\
\hline \multicolumn{6}{|c|}{ Utiliza medicamentos? * } \\
\hline Sim & $169(90,9)$ & $334(89.5)$ & $0,626^{\S}$ & 1,161 & $0,638-2,113$ \\
\hline Não & $17(9,1)$ & $39(10,5)$ & & 1,0 & \\
\hline \multicolumn{6}{|c|}{ № de medicamentos que utiliza * } \\
\hline Nenhum & $16(8,7)$ & $37(9,9)$ & $0,785^{\S}$ & 1,0 & \\
\hline De 1 a 3 & $61(33,2)$ & $130(34,9)$ & & 1,085 & $0,560-2,101$ \\
\hline >=4 medicamentos & $107(58,2)$ & $206(55,2)$ & & 1,201 & $0,639-2,258$ \\
\hline \multicolumn{6}{|l|}{ Escore do MEEM } \\
\hline Média $\pm D P$ & $22,3 \pm 5,0$ & $24,1 \pm 4,5$ & $<0,001^{€}$ & 0,931 & $0,898-0,966$ \\
\hline Amplitude & $7,0-30,0$ & $5,0-30,0$ & & & \\
\hline \multicolumn{6}{|c|}{ Presença de prejuizo cognitivo? * } \\
\hline Sim & $64(35,8)$ & $99(26,7)$ & $0,029^{\S}$ & 1,529 & $1,043-2,242$ \\
\hline Não & $115(64,2)$ & $272(73,3)$ & & 1,0 & \\
\hline \multicolumn{6}{|c|}{ Histórico de quedas * } \\
\hline Sim & $96(50,5)$ & $115(29,9)$ & $<0,001^{\S}$ & 2,398 & $1,675-3,432$ \\
\hline Não & $94(49,5)$ & $270(70,1)$ & & 1,0 & \\
\hline \multicolumn{6}{|c|}{ Queda no último ano * } \\
\hline Sim & $73(38,4)$ & $106(27,5)$ & $0,008^{\S}$ & 1,642 & $1,137-2,373$ \\
\hline Não & $117(61,6)$ & $279(72,5)$ & & 1,0 & \\
\hline
\end{tabular}

£: Nível mínimo de significância para a análise bivariada; OR: Odds Ratio; IC95\% Intervalo de confiança 95\% para o OR; *: dados apresentados na forma de n(\%); §: Teste Qui-quadrado de Pearson; €: Teste t-Student para grupos independentes assumindo heterogeneidade de variâncias.

Na Tabela 5 são apresentados o modelo inicial e o final da Regressão Logística Binária Multivariada aplicada para a predição do relato de perda urinária. Em função da perda do potencial preditor de determinadas variáveis, o modelo final foi composto por quatro variáveis, de forma que o grupo que relatou perda urinária pode ser predito pelas baixas pontuações no MEEM, pela presença de prejuizo cognitivo, por elevado tempo no teste da caminhada e pelo menor número de repetições no Teste Senta/Levanta. 
TABELA 5 - Modelos de Regressão Logística Binária Multivariada para a predição da incontinência urinária.

\begin{tabular}{|c|c|c|c|c|c|c|}
\hline \multirow{3}{*}{ VARIÁVEIS INDEPENDENTES } & \multirow{2}{*}{\multicolumn{2}{|c|}{$\begin{array}{l}\text { COEFICIENTE } \\
\text { DE REGRESSÃO }\end{array}$}} & \multirow[b]{3}{*}{ Sig. } & \multicolumn{3}{|c|}{ ODDS RATIO AJUSTADO } \\
\hline & & & & \multirow[b]{2}{*}{$\operatorname{Exp}(B)$} & \multicolumn{2}{|c|}{$\mathrm{IC} 95 \%$} \\
\hline & $B_{\text {bruto }}$ & S.E. & & & Inferior & Superior \\
\hline \multicolumn{7}{|l|}{ Modelo inicial } \\
\hline Sexo feminino & 0,065 & 0,234 & 0,780 & 1,067 & 0,675 & 1,687 \\
\hline Faixa etária - 70 a 79 anos & 0,016 & 0,233 & 0,074 & 1,117 & 0.944 & 1,605 \\
\hline Faixa etária - 80 anos ou mais & 0,236 & 0,449 & 0,059 & 1,090 & 0,928 & 1,904 \\
\hline Escore do MEEM menor & $-0,068$ & 0,030 & 0,026 & 0,935 & 0,881 & 0,992 \\
\hline Presença de prejuizo cognitivo & 0,483 & 0,297 & 0,075 & 1,128 & 0,944 & 1,805 \\
\hline IMC (maior) & 0,020 & 0,019 & 0,287 & 1,020 & 0,983 & 1,058 \\
\hline Maior tempo para caminhar $10 \mathrm{~m}$ & 0,138 & 0,064 & 0,032 & 1,148 & 1,012 & 1,301 \\
\hline Pior escore no teste Senta/levanta & $-0,066$ & 0,040 & 0,100 & 0,936 & 0,865 & 1,013 \\
\hline Queda no último ano & $-0,006$ & 0,224 & 0,980 & 0,994 & 0,641 & 1,542 \\
\hline \multicolumn{7}{|l|}{ Modelo final (5 etapas) } \\
\hline Escore do MEEM menor & $-0,006$ & 0,029 & 0,033 & 0,939 & 0,887 & 0,995 \\
\hline Presença de prejuizo cognitivo & 0,469 & 0,331 & 0,010 & 1,625 & 1,351 & 3,113 \\
\hline Maior tempo para caminhar 10 m & 0,469 & 0,062 & 0,016 & 1,160 & 1,028 & 1,309 \\
\hline Pior escore no teste Senta/levanta & $-0,069$ & 0,040 & 0,013 & 0,874 & 0,712 & 0,932 \\
\hline
\end{tabular}

IC95\%: Intervalo de Confiança 95\%; Bbruto: coeficiente de regressão bruto; S.E.: erro padrão para o coeficiente de regressão; Sig.: $\mathrm{p}$ = nivel minimo de significância para o coeficiente de regressão; Exp(B): Odds ratio; MEEM: Mini Exame do Estado Mental; IMC: indice de massa corporal. Modelo Inicial - Nota: R² de Nalgelkerke 0,314; Prova de Hosmer-Lemeshow (Qui quadrado = 9,662; $p=0,275$ ); Cox \& Snell: 0,285; Proporção geral de acerto - matriz de confusão: 66,3\%. Modelo Final - Nota: R² de Nalgelkerke 0,388; Prova de Hosmer-Lemeshow (Qui quadrado = 4,309; p=0,166); Cox \& Snell: 0,324; Proporção geral de acerto - matriz de confusão: 70,5\%.

\section{Discussão}

Conforme observado, neste estudo a prevalência da IU foi alta entre os idosos, principalmente entre as mulheres. É possivel que esses números reflitama realidade da cidade, posto que os dados coletados são provenientes de todos os distritos sanitários do municipio, e a distribuição da amostra do presente estudo, no que se refere ao gênero (mulheres= $64,3 \%$ ), foi similar à da população idosa total do municipio (mulheres=62,3\%), de acordo com o censo relativo ao período da coleta dos dados.?

Outro estudo, ${ }^{17}$ também realizado em Porto Alegre, apresentou prevalência na população estudada semelhante à do presente trabalho, onde $32,2 \%$ da população idosa da ESF de um único bairro relatou perda urinária. Resultados semelhantes de prevalência em idosos da ESF em São Paulo também foi relatada por Burti et al. ${ }^{18}$, dos quais $38,4 \%$ eram incontinentes. Esses achados não são restritos ao Brasil, pois há relatos na literatura internacional para todos os continentes que apontam para prevalências, em idosos que vivem em seus domicílios, que variam entre 2 e 41\% 4.5 , essa última próxima daquelas encontradas em Porto Alegre e São Paulo. ${ }^{77.18}$

O aumento da prevalência da IU com a idade 
e o fato de que ela acomete mais as mulheres são outros pontos em comum com achados na literatura nacional e internacional.45.19.20 $\mathrm{Na}$ presente amostra, enquanto $29,5 \%$ dos idosos de ambos os sexos até 69 anos relataram perda urinária, entre aqueles com 70 anos ou mais o relato foi feito por $38,9 \%$, e na reduzida amostra de individuos com 80 anos ou mais foi de quase $48 \%$.

Devemos considerar que, apesar de alta, a prevalência da IU pode ainda estar sendo subestimada devido à dificuldade das pessoas em procurar tratamento por motivos variados tais como se sentirem constrangidas, não considerarem o problema grave o suficiente para buscar assistência, medo de serem estereotipadas, acharem que é um aspecto normal do envelhecimento ou não terem conhecimento da possibilidade de tratamento. ${ }^{19}$ Em outros casos há o constrangimento por parte da mulher em compartilhar esse tipo de problema com os profissionais da saúde, principalmente os do gênero masculino. ${ }^{20}$ É possivel que uma mudança de atitude possa acontecer com medidas de educação em saúde que ajudem homens e mulheres a compreender o que é a IU e a buscar auxilio o mais cedo possivel, mas, principalmente, que possibilitem ações mais efetivas para a prevenção, detecção precoce e atendimentos desses casos na atenção básica. ${ }^{19}$ Para tanto, também se faz necessária a sensibilização das equipes da Atenção Primária quanto à importância da investigação rotineira dessa disfunção tão prevalente, que causa grande impacto negativo nos aspectos físico, psíquico, social e na qualidade de vida daqueles por ela acometidos, em particular os idosos. . $^{3.4}$

Além dos fatores previamente citados, a IU é um problema de saúde pública que gera gastos individuais e ao poder público. No Brasil, no ano de 2009, as mulheres com IU despenderam em média 4,7 salários mínimos nacionais da própria renda, desde o início dos sintomas até o encaminhamento para tratamento, enquanto - Sistema de Saúde despendeu 55\% de um salário mínimo por paciente para o diagnóstico e o tratamento. Esses custos tendem a aumentar com o crescimento da população idosa e a predominância do sexo feminino. ${ }^{21}$

O segundo objetivo do presente estudo foi determinar nesse grupo de idosos a relação da IU com dados sociodemográficos, funcionais e clínicos para a identificação dos fatores de risco para a população estudada, o que permitirá o planejamento de medidas e ações de promoção da saúde, uma vez que reconhecê-los e prevenilos pode reduzir a prevalência e a gravidade da incontinência. ${ }^{19}$ Assim, em meio a mais de 20 diferentes variáveis estudadas, foram identificados quatro fatores de risco para IU nos idosos da ESF/ POA: escores mais baixos no MEEM; presença de prejuizo cognitivo; e velocidade de caminhada mais lenta e menor força de membros inferiores.

Em relação aos escores mais baixos no MEEM, Quadros et al..22 também detectaram a mesma associação em estudo desenvolvido com idosos do interior de São Paulo. Esse achado, porém, não é restrito ao Brasil, e sim um problema mundial, conforme Drennan et al. ${ }^{23}$ relatam em sua revisão sobre o assunto.

No que tange o risco relativo para IU na presença de prejuízo cognitivo, Kessler et al.19 observaram que a população idosa incontinente tem maior prevalência de prejuizo cognitivo; mulheres e homens incontinentes têm, respectivamente, 1,2 e 1,6 vezes mais chance de prejuizo cognitivo, achado superior ao encontrado na presente amostra, que foi de 0,94 (Tabela 5). É possivel que o fato de Kessler et al. ${ }^{19}$ terem em sua amostra a maioria dos participantes com idade igual ou superior a 75 anos explique essa discrepância entre os dois valores para o risco relativo, posto que ambos fatores, IU ${ }^{3.4}$ e prejuizo cognitivo ${ }^{24}$ aumentam com o avanço da idade, inclusive conforme eles mesmos demonstraram em seus resultados.

Assim, como no presente estudo, Ruggero et al. ${ }^{25}$ também relataram associação significante entre IU e velocidade de caminhada mais lenta em uma amostra de 385 homens e de mulheres acima de 65 anos no estado de São Paulo, onde observaram odds ratio acima de 3.7 para velocidades de caminhada mais lentas nos idosos com IU, enquanto Fritel et al. ${ }^{24}$ reportaram odds ratio de 2,2 em uma amostra de 1.942 mulheres francesas acima de 75 anos. 
A velocidade da marcha está relacionada à força dos membros inferiores. ${ }^{26}$ Portanto, não surpreende o fato de que um menor escore do Teste Senta/Levanta, o qual reflete menor força de membros inferiores, seja também associado à IU e apontado como fator de risco para a mesma no presente estudo. Além disso, há evidências de que a musculatura pélvica reduz em conjunto com a massa muscular do corpo inteiro e que isso pode contribuir para a fisiopatologia da IU. ${ }^{27}$

Um dos fatores de risco para IU em mulheres amplamente apresentado na literatura é a obesidade/sobrepeso. ${ }^{28}$ Entretanto, diferente da grande maioria dos estudos, em nossos dados essa relação não foi observada. É possivel que os nossos achados difiram da literatura sobre o assunto em razão da amostra apresentar um percentual considerável de homens (35,6\%), bem como o fato de que os individuos com IU não diferiram estatisticamente daqueles sem IU em termos de peso, componente do IMC. A outra medida que forma o IMC, a altura, diferiu estatisticamente, contudo, acreditamos que a diferença - 2 cm - não tem importância clínica ou funcional. Ademais, ao se observar a distribuição da amostra em categorias do IMC, observa-se que individuos com e sem IU não diferiram, tendo os dois grupos, portanto, percentuais semelhantes de individuos com obesidade/sobrepeso.

O desenho transversal do estudo, com apenas um ponto de medição, é uma limitação, uma vez que a direção da relação causal entre IU e os fatores de risco não puderam ser determinados, pois essa associação também pode ser bidirecional. Outra limitação se deve à logística de acesso à população estudada, que fez com que a presença de doenças fosse avaliada por autorrelato, o que pode resultar em sobrestimação ou subestimação da prevalência da doença. O fato de a IU ter sido avaliada apenas por questionário pode ser considerada outra limitação do estudo. Sendo um estudo epidemiológico, seria financeiramente inviável que a incontinência fosse clinicamente confirmada. Ademais, a definição escolhida para IU está de acordo com as diretrizes da Sociedade Internacional de Continência para a sua investigação. ${ }^{29}$
Há que se ressaltar a representatividade da amostra investigada, de tamanho suficiente para um estudo de prevalência, selecionada de forma aleatória e proporcional em todos os distritos sanitários do município, o que pode ter reduzido o viés de seleção.

As autoridades e os profissionais de saúde poderão utilizar os dados aqui apresentados para o planejamento de ações e de estratégias que favoreçam a promoção da saúde dessa população fragilizada pelo envelhecimento, baixa escolaridade. Principalmente no caso daqueles com IU, maior prejuízo cognitivo e funcional, os quais, provavelmente, convivem com o estigma associado à perda involuntária de urina, apresentam pior qualidade de vida e custos de saúde mais altos. ${ }^{3-5,21}$

\section{Conclusão}

Baseados nos resultados apresentados, podemos concluir que, na amostra populacional estudada, foi alta a prevalência da IU, sendo identificados como fatores de risco para essa condição a menor força de membros inferiores, a velocidade de caminhada mais lenta e o prejuizo cognitivo, todos modificáveis.

\section{Referências}

1. Silva JVF, Silva ED, Rodrigues APRA, Miyazawa AP. "A relação entre o envelhecimento populacional e as doenças crônicas não transmissiveis: sério desafio de saúde pública." Cad Grad Ciên Bio Saúde. 2015; 2(3):91-100.

2. Silva FR; Gardenghi G; Conceição PO. Perfil de idosos submetidos à avaliação geriátrica ampla em Serviço de reabilitação. Rev Bras Promoç Saúde. 2017:30(2):170-78. https://doi. org/10.5020/18061230.2017.p170.

3. Alvarenga-Martins N, Pinto PF, Arreguy-Sena C, Paschoalin, HC; Moura, DCA; Teixeira, CV. Incontinência urinária: uma análise à luz das políticas de envelhecimento. Rev enferm UFPE on line. 2017:11(3):1189-99.

4. Gorina Y, Schappert S, Bercovitz A, Elgaddal N, Kramarow E. Prevalence of incontinence among older americans. National Center for Health Statistics. Vital Health Stat. 2014:3(36):1-33.

5. Carneiro JA, Ramos GCF, Barbosa ATF, Medeiros SM, Lima CA, Costa FM. et al. Prevalência e fatores associados à incontinência urinária em idosos não institucionalizados. Cad. Saúde Colet. 2017;25 (3): 26877. https://doi.org/10.1590/1414-462×201700030295. 
6. DATASUS. [capturado em 3 Ago 2019]. Disponível em: www.datasus.gov.br.

7. IBGE - Instituto Brasileiro de Geografia e Estatística. Censo Demográfico 2010. [capturado em 3 Ago 2019]. Disponivel em: http://cens02010.ibge.gov.br/.

8. Brasil. A Implantação da Unidade de Saúde da Familia. Costa Neto MM (org). Brasilia: Ministério da Saúde, Secretaria de Políticas de Saúde, Departamento de Atenção Básica; 2000.

9. Gomes I, Nogueira EL, Engroff P, Ely LS, Schwanke CHA, Carli GA. et al. The multidimensional study of the elderly in the Family Health Strategy in Porto Alegre, Brazil (EMI-SUS). PAJAR. 2013;1(1):20-24.

10. World Health Organization. Obesity: preventing and managing the global epidemic (report of a WHO consultation on obesity). Genebra: World Health Organization, 1997.

11. Rikli RE, Jones J. Teste de Aptidão Física para Idosos. Barueri, SP: Manole; 2008.

12. Matsudo, SM. Avaliação do idoso: física \& funcional. 2. ed. Londrina: Midiograf; 2005

13. Fess, EE. Grip strength. In: Casanova JS, editor. In Clinical assessment recommendations. 2 a ed. Chicago: American Society of Hand Therapists; 1992. p. 41-45

14. Bertolucci PHF, Brucki SMD, Campacci S, Juliano Y. O Mini-Exame do Estado Mental em uma população geral: impacto da escolaridade. Arq Neuropsiquiatr 1994:52:1-7. https://doi.org/10.1590/S0004-282X1994000100001.

15. Scagliusi FB, Alvarenga M, Polacow VO, Queiroz, GKO, Coelho D, Philippi, A. et al. Concurrent and discriminate validity of the Stunkard's figure rating scale adapted into Portuguese. Appetite. 2006; 47(1):77-82. https://doi.org/10.1016/i.appet.2006.02.010.

16. Sardinha A, Levitan MN, Lopes FL, Perna G, EsquiveL G, Griez EJ et al. Tradução e adaptação transcultural do questionário de atividade física habitual. Rev Psiq Clín. 2010;37(1):16-22. https://doi. org/10.1590/S0101-60832010000100004.

17. Rosa LHT, Souza CM, Lima CHL, Boggio ESB, Santos FC, Carboni $\mathrm{C}$. et al. Prevalência da incontinência urinária em idosos de Porto Alegre-RS. Rev Geriat Gerontol. 2014; 8(2): 104-09.

18. Burti JS, Santos AM, Pereira RM, Zambon JP, Marques AP. Prevalence and clinical characteristics of urinary incontinence in elderly individuals of a low income. Arch Gerontol Geriatr. 2012;54(2):e42-46. https://doi.org/10.1016/j.archger.2011.04.004.

19. Kessler M, Facchini LA, Soares MU, Nunes BP, França SM, Thumé E. Prevalência de incontinência urinária em idosos e relação com indicadores de saúde física e mental. Rev Bras Geriatr Gerontol. Rio de Janeiro, 2018; 21(4): 409-419. https://doi. org/10.1590/1981-22562018021.180015.
20. Marques LP, Schneider IJC, Giehl MWC, Antes DL, d'Orsi E. Demographic, health conditions and lifestyle factors associated with urinary incontinence in elderly from Florianopolis, Santa Catarina, Brazil. Rev Bras Epidemiol.2015;18(3):595-606. https://doi. org/10.1590/1980-5497201500030006.

21. Araújo GT. O custo da incontinência urinária no Brasil experiência do serviço de Uroginecologia da UNIFESP [dissertação de mestrado]. São Paulo: Programa de Pós-graduação em Ciências da Saúde, Escola Paulista de Medicina, Universidade Federal de São Paulo; 2009

22. Quadros LB, Aguiar A, Menezes AV, Vieira, A, Alves $E F$, Nery T. et al. Prevalência de incontinência urinária entre idosos institucionalizados e sua relação com o estado mental, independência funcional e comorbidades associadas. Acta Fisiatr. 2015;22(3):130-34.

23. Drennan VM, Cole L, Rait G, Cole L, Grant R, lliffe S The prevalence of incontinence in people with cognitive impairment or dementia living at home. Neurourol Urodyn. 2013:32: 314-24. https://doi.org/10.1002/nau.22333.

24. Fritel $X$, Lachal L, Cassou B, Fauconnier A, Dargent-Molina P. Mobility impairment is associated with urge but not stress urinary incontinence in community-dwaelling older women: Results from the Ossebo study. BJOG 2013;120:1566-72. https:// doi.org/10.1111/1471-0528.12316.

25. Ruggero CR, Bilton TL, Teixeira LF, Ramos, JLA, Alouche SR, Dias RC, Perracini MR. Gait speed correlates in a multiracial population of community-dwelling older adults living in Brazil: a cross-sectional population-based study. BMC Public Health. 2013;28(13):182. https://doi.org/10.1186/1471-2458-13-182..

26. Bianchi AB, Oliveira JM, Bertolini SMMG. Marcha no processo de envelhecimento: alterações, avaliação e treinamento. Rev Uningá, 2015;45:52-55.

27. Langoni CS, Knorst MR, Lovatel GA, Leite VO, Resende TL. Urinary incontinence in elderly women from Porto Alegre: its prevalence and relation to pelvic floor muscle function. Fisioter. Pesqui. 2014;21(1):74-80 https://doi.org/10.1590/1809-2950/475210114.

28. Menezes M, Pereira M, Hextall A. Predictors of female urinary incontinence at midlife and beyond. Maturitas. 2010 Feb;65(2):167-71. https://doi.org/10.1016/j.maturitas.2009.10.004.

29. ICS. International Continence Society. Current definitions. [cited 2019 Abr 14]. Available from: https:// www.ics.org/terminology/113.

\section{Vivian Ulrich}

Especialista em Atenção Geriátrica Integrada pela Pontifícia Universidade Católica do Rio Grande do Sul (PUCRS, Porto Alegre, RS, Brasil).

\section{Raquel Rousselet Farias}

Especialista em Acupuntura pelo Centro Universitário Leão Sampaio de Juazeiro do Norte (UNILEÃO, Juazeiro do Norte, CE, Brasil). 


\section{Mara Regina Knorst}

Doutora em Gerontologia Biomédica, Pontificia Universidade Católica do Rio Grande do Sul (PUCRS, Porto Alegre, RS, Brasil).

\section{João Henrique Corrêa Kanan}

Doutor em Bioquímica e Biologia Molecular Aplicada pela Universidade Federal do Rio Grande do Sul (UFRGS, Porto Alegre, RS, Brasil).

\section{Thaís de Lima Resende}

Doutora em Ciências da Saúde pela Pontificia Universidade Católica do Rio Grande do Sul (PUCRS, Porto Alegre, RS, Brasil), professora da Escola de Ciências da Saúde e da Vida (PUCRS, Porto Alegre, RS, Brasil).

\section{Endereço para correspondência}

Thais de Lima Resende

Pontificia Universidade Católica do Rio Grande do Sul

Av. Ipiranga, 6681, Prédio 81, $6^{\circ}$ andar, Sala 603

Partenon, 90619-900

Porto Alegre, RS, Brasil 